Dear Professor Buchsbaum,

We are pleased to submit a revised version of our manuscript titled Social anhedonia and asociality in psychosis revisited. An experience sampling study in which we complied with all format changes to the best of our ability.

Thank you for the acceptance of our manuscript for publication in Psychiatry Research.

Sincerely,

Zuzana Kasanova, on behalf of all co-authors

Contact information:

Zuzana Kasanova, PhD

KU Leuven, Department of Neuroscience

Kapucijnenvoer 35, Leuven, 3000 Belgium

zuzana.kasanova@kuleuven.be 


\section{Highlights}

Patients with psychosis match controls in time spent in unstructured social context

Patients spend less time in structured social context than the controls do

Patients report intact hedonic experience of both social contexts

Patients' lifestyle and avolition symptoms predict time spent in both social contexts 


\title{
Social anhedonia and asociality in psychosis revisited. An experience sampling study
}

\author{
Zuzana Kasanova ${ }^{\mathrm{a}^{*}}$, Margreet Oorschot ${ }^{\mathrm{b}}$, Inez Myin-Germeys ${ }^{\mathrm{a}}$,
}

\begin{abstract}
The DSM-5 appended the conceptualization of asociality in psychotic disorders as the manifestation of diminished interest in social interactions, but it also admitted that it might merely be the result of limited opportunities for social interactions. In an effort to investigate this apparent dichotomy, we used experience sampling data from 149 patients with psychotic disorder and 143 controls, and divided their social interactions into those occurring in the context of work and other structured activities that patients have limited access to, and those occuring in the context of unstructured activities such as visits and conversations that both groups can choose relatively more freely. Patients spent significantly smaller proportion of their time in structured social context, but matched the controls in the time spent in unstructured social contexts, and endorsed intact hedonic experience of both social contexts. Additionally, employment and living situation, rather than the severity of negative symptoms, predicted the proportion of time patients spent in unstructured social contexts, suggesting a lack of evidence for asociality in contexts where patients have equal opportunities for social interactions.
\end{abstract}

Keywords: Psychosis, Negative symptoms, Asociality, ESM, EMA 
Social anhedonia and asociality in psychosis revisited. An experience sampling study

$$
\text { Zuzana Kasanova }{ }^{a^{*}} \text {, Margreet Oorschot }{ }^{\mathrm{b}} \text {, Inez Myin-Germeys }{ }^{\mathrm{a}} \text {, }
$$

${ }^{a}$ Center for Contextual psychiatry, Department of Neurosciences, KU Leuven- Leuven University, Leuven, Belgium

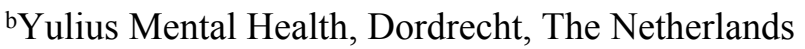

*Corresponding author: Zuzana Kasanova, Kapucijnenvoer 33, blok I, 3000 Leuven zuzana.kasanova@kuleuven.be 


\begin{abstract}
The DSM-5 appended the conceptualization of asociality in psychotic disorders as the manifestation of diminished interest in social interactions, but it also admitted that it might merely be the result of limited opportunities for social interactions. In an effort to investigate this apparent dichotomy, we used experience sampling data from 149 patients with psychotic disorder and 143 controls, and divided their social interactions into those occurring in the context of work and other structured activities that patients have limited access to, and those occurring in the context of unstructured activities such as visits and conversations that both groups can choose relatively more freely. Patients spent significantly smaller proportion of their time in structured social context, but matched the controls in the time spent in unstructured social contexts, and endorsed intact hedonic experience of both social contexts. Moreover, employment and living situation, in addition to the severity of symptoms of avolition, predicted the proportion of time patients spent in structured and unstructured social contexts, supporting the notion that both lifestyle as well as disease-specific factors contribute to real-life social behavior in psychosis.
\end{abstract}

Keywords: Psychosis, Negative symptoms, Asociality, ESM, EMA 


\section{Introduction}

The DSM-5 lists negative symptoms among the key features that define psychotic disorders (American Psychiatric Association, 2013). These include diminished emotional expression, avolition, alogia and anhedonia. Asociality assumes a somewhat ambiguous status, as it "...refers to the apparent lack of interest in social interactions and may be associated with avolition, but it can also be a manifestation of limited opportunities for social interaction.”(American Psychiatric Association, 2013) Such dichotomous definition of asociality as the consequence of avolition or of a socially passive lifestyle of individuals with psychosis, reflects the lack of consensus on concepts and assessments of asociality (Marder and Galderisi, 2017), as well as the paradoxical findings from the literature. Specifically, confluent neuroimaging (Dowd and Barch, 2010) and physiological evidence (Horan et al., 2010) shows intact hedonic experience of pleasant stimuli among patients with schizophrenia (for meta-analyses see (Cohen and Minor, 2010; Yan et al., 2012), especially if these have a social content (Bodapati and Herbener, 2014). Nonetheless, patients consistently exhibit deficits in anticipating pleasurable events and seeking them out (Marder and Galderisi, 2017).

Particularly revealing is evidence from experience sampling studies where the same sample of patients with schizophrenia endorsed appropriate in-the-moment hedonic experience of pleasant events and social company, but diminished goal-directed behavior (Gard et al., 2007) and time spent in the company of others (Collip et al., 2014). This observation of decoupling between hedonic experience and behavior has been confirmed experimentally using social and non-social evocative stimuli, with reports of intact ability to experience their pleasantness, in the presence of reduced drive to gain access to them among individuals with psychosis (Heerey and Gold, 2007; Lui et al., 2016; Xie et al., 2017). In other words, even though the emotional experiences are consistently reported to be relevant for patients, they do not seem to suffice in guiding the pertinent behavior.

One often overlooked caveat of the empirical and experience sampling approaches to the study of hedonism and volition, however, is the substantial inequality in the environments that individuals with psychosis and the comparison subjects are exposed to and interact with. Concretely, it is estimated that up to $90 \%$ of adults with psychotic disorder are unemployed and 60\% is single (Olsson et al., 2016; Oorschot et al., 2013). The practical implications with respect to the goal-directed and social activities are that while controls tend to spend the bulk of their day attending to obligations such as work meetings, commuting, childcare etc., patients have been found to spend a large portion of their time engaged in solitary, passive activities, such as sleeping, watching TV or listening to the radio (Eklund et al., 2009). While 
it has been thought that such profound differences in patients' lifestyle are driven by anhedonia and asociality (Rocca et al., 2014), one could also reason that fewer imposed obligations drive the findings of decreased social behavior. Indeed, when patients were compared to controls on activities that both groups could choose more freely, such as entertainment, sport, learning and applying knowledge, they endorsed limited diversity, but similar frequency of these behaviors (Lipskaya-Velikovsky et al., 2016). Gard and colleagues (Gard et al., 2014a) extended these results by reporting that patients engaged in similar amounts of activities and planned similar number of goals throughout their days as the comparison subjects, but their activities and goals tended to be significantly more pleasurebased and require less effort. It is especially noteworthy that patients exceeded the controls' ratings of pleasantness of their anticipated social activities, and yet endorsed significantly fewer social goals (Gard et al., 2014a).

In conclusion, it remains unclear whether the paradoxical findings of limited social engagement in the presence of intact hedonic experience of social company reflect true generalized asociality, or whether they are an artifact of a lifestyle in which patients tend to have lesser access to social company encountered in the context of work and family obligations. This distinction has important ramification for the design of effective interventions aimed at social and occupational functioning of individuals with psychosis. We therefore revisited the experience sampling data indicating intact hedonic experience of, and time spent in, social company in patients with psychosis (Oorschot et al., 2013). To systematically address the question whether social behavior and hedonic experience change as a function of opportunities for social interactions as well as avolition, we first divided all social contexts into i) being alone; ii) structured company that is encountered during work, school, childcare, etc., to which patients tend to have limited access, iii) unstructured social contexts, such as visiting others, and going to the movies, that patients should theoretically have relatively more access to, and iv) other social contexts that can not be classified as either structured or unstructured.. We then assessed the behavioral manifestation of asociality by comparing patients to controls on the proportion of time spent in each social context per assessment period. To test differences in hedonic experience of each context, we compared the two groups on the amount of positive affect endorsed in structured and unstructured company, versus being alone. Subsequently, we assessed the impact of the severity of avolition and the contribution of lifestyle to these daily-life indices of social behavior.

\section{Methods}




\subsection{Sample}

Data from 143 healthy controls and 149 patients with a diagnosis of schizophrenia spectrum disorder who completed a battery of lifestyle, demographic and clinical assessments, as well as experience sampling self-assessments are presented in this study (Oorschot et al., 2013). Lifestyle information was missing from one patient and two control participants. The general inclusion criteria were age between 18 and 70 years and sufficient command of the Dutch language to understand the instructions and questionnaires. Additional inclusion criterion for the patient group was the diagnosis of schizophrenia, schizoaffective disorder or schizophreniform disorder, as confirmed by the Comprehensive Assessment of Symptoms and History (Andreasen et al., 1992). General exclusion criteria consisted of brain disease and a history of head injury with loss of consciousness. Healthy controls were excluded if presenting with a lifetime or family history of the schizophrenia spectrum disorder. Patients were recruited via flyers from mental health facilities in the Netherlands and in Flanders, Belgium. Controls were recruited from the same regions through newspaper advertisements or random mailings. Detailed demographic composition of both samples is presented elsewhere (Oorschot et al., 2013) and information relevant for the current analyses also in table 1.

\subsection{Procedures}

This study combines data from three experience sampling studies conducted in the department of Psychiatry and Neuropsychology of the Maastricht University, with information detailing the study procedures published elsewhere (Oorschot et al., 2013). Briefly, the momentary assessments used in the present analyses overlapped entirely across studies and were collected 10 times per day on six consecutive days. All participants were provided with a digital wristwatch and six booklets, each containing 10 assessments to be filled out every day. The wristwatches were programmed to emit 10 signals ("beeps") at an unpredictable moment between 7:30 and 22:30, on average once every 90 minutes. Each beep was a prompt for the participant to fill out the self-assessments, consisting of several items endorsing the current affect, activity and context on a 7-point Likert scale ( $1=$ not at all to $7=$ very much) (Oorschot et al., 2013).

\subsection{Assessments}

\subsubsection{Lifestyle indicators}

Demographic information pertaining to vocation and living situation was used to construct four different lifestyle categories, each reflecting an environment that is conducive to a certain 
level of social contexts the participant is likely to be exposed to on a moment-to-moment basis: 1. no vocational activities (permanent disability/ long-term disability/unemployed) and no cohabitation (living alone); 2. no vocational activities and cohabitating (living with housemates/roommates, parents or other family, partner, own children); 3. vocational activities (full-time/part-time employment/ volunteer work/sheltered work, attending school at any level) and no cohabitation; 4 . vocational activities and cohabitating.

\subsubsection{Symptoms of psychosis}

The severity of symptoms of psychosis was ascertained using the positive and negative syndrome scale (PANSS) with a reference period of 2 past weeks. All items were rated on a 7-point scale ( $1=$ absent to 7=extreme). We employed the well-validated 5-factor solution of the PANSS scale (van der Gaag et al., 2006; Woodward et al., 2014), to construct the positive symptoms, disorganization, excitement and emotional distress score by summing the scores on the pertinent items (van der Gaag et al., 2006). The negative symptom subscale has since been further re-conceptualised into a two-factor solution that differentiates between expressive deficits dimension, such as alogia, and avolition dimension (Fervaha et al., 2014; Jang et al., 2016). The avolition dimension includes two items that specifically assess social avolition, and has been shown to be more closely linked to social functioning (Marder and Galderisi, 2017), and predict social contacts over a 5-year period, than the other negative symptom factors (Galderisi et al., 2013). We therefore expected it to uniquely relate to social behavior in the daily life, and in all analyses focused specifically on the avolition dimension of the negative symptomatology. We constructed it by summing the 3 PANSS items: emotional withdrawal, passive social withdrawal, active social avoidance)(Jang et al., 2016). Internal consistency of the resulting avolition construct was satisfactory (Cronbach's $\alpha=$ $0.84)$.

\subsubsection{Experience sampling data}

\subsubsection{Momentary positive affect}

Positive affect (PA) was assessed using three adjectives rated on a 7-point Likert scale: 'I feel cheerful', 'I feel content', 'I feel relaxed'. The choice of these items reflected results from previous ESM studies that selected items with high loadings on PA latent factor and sufficient within-person variability, and was confirmed by a factor analysis performed on the current data (Cohen's $\alpha=0.73$ ). 


\subsubsection{Social contexts}

At each measurement moment the participants were asked to indicate whether they were presently in the company of other people by responding to the question 'Who are you with (just before the beep)?. During the briefing procedure, participants were instructed to indicate that they were alone when they subjectively perceived to be alone, even though there were, strictly speaking, other people on the street or in the house. Similarly, participants were instructed to fill out that they were in the company when they subjectively perceived the company of others. For the purposes of the current study, we later assigned the score of 1 to being in company and 0 to being alone.

Similarly, participants also had to indicate what their current activity was, by responding to the question 'What are you doing (just before the beep)?', which we later categorized into work, passive-leisure, self-care, transportation etc. From these, the following 11 categories were considered to be a goal-directed activity based on previous research (Oorschot et al., 2013): work, volunteer work, study, household chores, childcare, care to adults, shopping and services, personal medical care, personal hygiene, related transport, political/civic activity. If a participant indicated to be in the company of others and at the same time engaged in a goaldirected activity, we operationalized it as being in structured social context. An example of such activity could involve driving a child to school, participating in a work meeting, etc. This category was constructed in order to differentiate contexts to which healthy controls tend to have easier access to, simply by virtue of being more likely to participate in goal-directed vocational activities and family obligations in which they encounter the company of others. The following 7 categories of activities were classified as non-goal-directed: conversation, online social contact, parties, visits, cafe/pub, movies/theatre, other leisure activities, physical interaction. If a participant indicated to be in the company of others and at the same time in one of these non-goal-directed activities, we conceptualized it as unstructured social context. All other activities that could not be classified as either unstructured or structured, but were done in the company of others, were classified as other social contexts.

\subsubsection{Operational definition of hedonic experience}

We conceptualized hedonic experience as the extent to which subjective positive affect (PA) is associated with being engaged in the current social context. That is, hedonic experience of structured social context was defined as its pairing with the ratings of PA. Similarly, the hedonic experience of unstructured social context was defined as its association with PA. 


\subsection{Statistical analyses}

All analyses were conducted using STATA 14.2 (StataCorp). For analyses using solely timeinvariant variables: group, demographic variables and clinical symptom scores, we applied linear and logistic regressions. First, we compared the patient and control groups on lifestyle by entering it as an outcome of ordinal regression with group as the predictor. Due to the unbalanced distribution of lifestyle categories among patients compared to controls, lifestyle was further used only in within-group analyses of the patient sample. We then performed a multinomial logistic regression in the patient group only, with avolition as the predictor and the four levels of lifestyle as the outcome variable, with the most common lifestyle $(B=$ no vocational activities, cohabitating) as the reference category, while controlling for all other PANSS symptom domains.

Multilevel mixed regressions were used for the ESM observations in order to model the nested data: within-subject momentary ratings at every beep (level 1) are nested within individuals comprising the between-group variable (controls, patients with psychosis; level 2) (Myin-Germeys et al., 2009; Oorschot et al., 2012). The structure of the matrix that takes the covariation of within-subject observations into account is covariance unstructured, and was thus used in all analyses.

\subsubsection{Proportion of time spent in social contexts}

The proportion of time spent in each social context was entered into a multilevel mixed regression analysis as the outcome variable, and group as the predictor.

In order to test whether patients' lifestyle was associated with the proportion of time they spent in structured and unstructured social contexts, it was entered as a predictor into the multilevel mixed regression, with the proportion of time spent in structured/unstructured social context as the separate outcome variable. The same procedure was then repeated with PANSS avolition score as a predictor, and proportion of time spent in the social context as the outcome variable, while controlling for all other PANSS symptom domains.

\subsubsection{Hedonic experience of social contexts}

A series of multilevel mixed regression analyses were conducted with PA as the outcome variable and the particular social context, group and their interaction as the predictors. In order to only test the hedonic experience at the current moment, we controlled for lagged PA at the previous measurement moment. To test whether patients' lifestyle moderated the relationship between the social context and PA (i.e. hedonic experience), we performed a 
multilevel mixed regression with lifestyle as the predictor in interaction with structured/ unstructured social contexts and PA as the outcome variable. We repeated the same procedure with PANSS avolition score as a predictor in interaction with structured/unstructured social contexts, and PA as the outcome variable, while controlling for all other PANSS symptom domains.

\section{Results}

\subsection{Demographic, lifestyle and clinical symptoms}

As reported previously (Oorschot et al., 2013) and here also presented in table 1, the patient group was significantly younger (by approximately one year), and included a significantly higher proportion of men compared to the control group. We therefore controlled for age and gender in all analyses. In terms of lifestyle, relative to the comparison subjects, patients were significantly less likely to have a lifestyle characterized by no vocational activities while cohabitating, and significantly less likely to have a lifestyle with vocational activities and cohabitating ( figure 1a, 1b). Importantly, the patients' PANSS avolition score did not influence the odds of having a lifestyle different from the most common one of cohabitation without vocational activities (all p-values $>0.05$; detailed results in Supplementary table 1).

\subsection{Time spent in social contexts}

During the assessment week, relative to healthy volunteers, patients with psychosis were found to spend a significantly smaller proportion of time in structured social contexts, but matched the controls on the proportion of their time spent in unstructured social contexts, as well as time spent alone (Table 1)

3.2.1 The association between lifestyle and proportion of time spent in social contexts Table 2 demonstrates that in the patient group, the different lifestyles were differentially associated with the proportion of time spent in social contexts. Specifically, those who cohabitated and participated in vocational activities spent significantly higher proportion of their time in structured social contexts than those with all other lifestyles (contrasts D vs. A, $\mathrm{B}, \mathrm{C}$ in table 2). Additionally, even without vocational activities, those who cohabitated spent significantly higher portion of their time in structured social contexts than those who lived alone (contrast A vs. B in table 2).

In terms of higher proportion of time spent in unstructured social contexts, the only significant difference emerged between the lifestyle of cohabitation with vocational activities (D) and 
lifestyle of no cohabitation and no vocational activities (A). Again, even without vocational activities, those who cohabitated spent significantly higher proportion of their time in unstructured social contexts than those who did not (contrast A vs. B, table 2).

\subsubsection{The association between avolition and proportion of time spent in social contexts} In the patient group, the severity of symptoms of avolition was significantly negatively associated with proportion of time spent in structured social contexts $[\mathrm{B}=-0.12, z=-3.02$, $p=0.003, \mathrm{CI}(-0.2,-0.04)]$. as well as unstructured social contexts $[\mathrm{B}=-0.13, z=-3.2, p=0.001$ $\mathrm{CI}(-.2,-.05)]$.

3.2.3 The relative contribution of lifestyle versus avolition to proportion of time spent in social contexts

Since both lifestyle and the severity of avolition appeared to predict the proportion of time spent in social contexts, we tested their specific contribution in a single multilevel regression with two predictors: lifestyle as a categorical variable, and a standardized PANSS avolition score as the predictors. This approach allowed us to compare the two predictors measured on different scales, since for both a one unit change indicates a one standard deviation increase in their influence on the outcome variable. This test revealed that while both predictors have a significant, though opposing, impact on the time spent in structured social contexts, a one unit change in lifestyle (from A to B to C to D) increased the proportion of time spent in structured social contexts by $45 \%[z=3.63, \mathrm{p}<0.001, \mathrm{CI}(0.21,0.7)])$, and a one unit increase in avolition decreased the proportion of time spent in structured social contexts by $24 \%[z=-2.07$, $p=0.038, \mathrm{CI}(-0.46,-0.01)]$.

Similarly, lifestyle had a relatively stronger impact on time spent in unstructured social contexts, with one unit change in lifestyle (from A to B to $\mathrm{C}$ to $\mathrm{D}$ ) increasing the proportion of time spent in unstructured social contexts by $35 \%[z=2.70, p=0.007, \mathrm{CI}(0.09,0.6)]$, whereas only a trend emerged for one unit increase in avolition score predicting a decrease in time spent in unstructured social contexts by $22 \%[\mathrm{z}=-1.86, p=0.062, \mathrm{CI}(-0.45,0.01)]$.

\subsection{Hedonic experience of social contexts}

As shown in table 3, neither group endorsed significant increase in PA in structured social contexts, with no group x structured social context interaction on ratings of PA. Contrastingly, unstructured social contexts were associated with significant rise in PA in both groups equally (table 3). 
Importantly, no significant group x social context interaction on PA levels emerged when the structured and unstructured social contexts were compared to each other $[\mathrm{B}=0.05, z=0.65$, $p=0.518, \mathrm{CI}(-0.1,0.2)]$, with patients reporting a significant increase in PA in unstructured compared to structured social contexts $[\mathrm{B}=0.17, \mathrm{z}=2.61, p=0.009, \mathrm{CI}(0.04,0.31)]$ that was matched by that of the controls $[\mathrm{B}=0.22, z=5.05, p=<0.001, \mathrm{CI}(0.14,0.31)]$.

\subsubsection{The relationship between lifestyle and hedonic experience of social contexts}

Within the patient group, lifestyle did not moderate the relationship between structured social contexts and PA (i.e. hedonic experience), nor between the unstructured social context and PA (all p-values $>0.05$ ).

3.3.2 The relationship between avolition symptoms and hedonic experience of social contexts Within the patient group, PANSS avolition score did not moderate the relationship between structured social contexts and PA (i.e. hedonic experience) $[\mathrm{B}=-0.03, z=-1.37, p=.0170, \mathrm{CI}(-$ 0.07, 0.01)], but did emerge as a significant moderator of the relationship between the unstructured social context and $\mathrm{PA}[\mathrm{B}=-0.07, z=-2.94, p=0.003, \mathrm{CI}(-0.12, p=-0.02)]$. That is, the patients who scored higher on the avolition factor endorsed lower PA in unstructured social contexts relative to those who scored lower on avolition severity.

\section{Discussion}

This study employed experience sampling method to investigate how individuals with psychosis allocate their time to various social contexts, and assess their hedonic experience of them. Patients spent significantly smaller proportion of their time in structured social context, but matched the controls in the time spent in unstructured social contexts. Additionally, as expected based on findings of no generalized hedonic deficit in psychosis (Oorschot et al., 2013), patients endorsed intact hedonic experience of both social contexts. Patients' lifestyle and symptoms of avolition were not only found to be unrelated to each other, but they were also found to have a differential impact on their time spent in social contexts and the hedonic experience thereof.

\section{Structured social contexts}

The assessments of the structured social contexts revealed rather expected evidence for the anhedonia paradox: patients spent smaller proportion of their time in this company despite their intact hedonic experience of it (Fortunati et al., 2015; Strauss, 2013). This finding aligns with emerging experimental evidence for a disconnect between self-reported hedonics and the 
corresponding motivated behavior in schizophrenia spectrum disorders (Heerey and Gold, 2007; Lui et al., 2016). Not surprisingly, in the current study lifestyle and avolition symptoms were both associated with the time patients spent in structured social contexts, with lifestyle emerging as a relatively stronger predictor of the time spent in this context. Neither the patients' lifestyle nor the severity of avolition, however, were related to the hedonic experience of this context,. That is, diminished engagement in structured social interactions, but not the hedonic experience thereof, appears to be a reflection of lifestyle in addition to the reduced social volition dimension of the negative symptomatology of the disorder.

\section{Unstructured social contexts}

Novel insights can be derived from our analyses of the unstructured social contexts, that revealed a rare instance of intact engagement in social behavior being found in the psychosis group. Although no group differences were detected, patients spent nearly $22 \%$ of the assessment week in unstructured social contexts, while the controls spent approximately $20 \%$ of their time in this context. Along with the finding that patients matched the controls in their positive hedonic experience of the unstructured social contexts, these results align with evidence for preserved volition for interpersonal connection in this group (Gard et al., 2014b). Importantly, the patients who lived with others and participated in vocational activities tended to spend greater proportion of their time in this context, whereas those with lower symptoms of avolition tended to have higher hedonic experience of it.

Despite the surprising gap in our knowledge of patients' engagement with their real-world environments, important insights can be derived from a meticulous study by Gard and colleagues in which researchers called patients with psychosis and healthy controls several times a day to inquire about their goals and activities (Gard et al., 2014a). The authors observed that patients surpassed the healthy controls in the amount of pleasure-based activities, and matched their hedonic experience of these activities, irrespective of the unemployment rate. This set of findings fully supports our observations from unstructured social contexts. One is then compelled to ponder why it is that patients seem to show intact experience of, and engagement in unstructured social contexts, but show marked deficits in the time they devote to structured social contexts.

One plausible explanation for the discrepant engagement in social contexts is that each might require different effort; Simply put, in order to become engaged in the company of people encountered during goal-directed activities, one must first go through several steps in order to gain access to those activities, such as qualification classes and job training, applying for school admission or a job, going through the interview process, etc. Indeed, while unexplored 
in the present study, Gard and colleagues (2014) reported that patients tended to be engaged in, and plan less effortful activities. Likewise, in a task that required minimal effort, patients with psychosis demonstrated unimpaired preference for and volition towards rewarding stimuli (Suri et al., 2018). High rewards did not appear to sufficiently incentivize patients' behavior, however, when the effort required was also high (Gold et al., 2013; Gold et al., 2015; McCarthy et al., 2016). On the other hand, one could also argue that from a short-term perspective, attendance of family and work-related social interactions might require relatively less effort as they tend to be pre-scheduled and often initiated by others, as opposed to conversations and pub visits that might require active participation and initiation. Further research is necessary to disentangle the putative asymmetry in effort allocation to these two contexts.

Another hypothetical explanation for our findings of contrasting patterns of patients' engagement in structured and unstructured social contexts is societal and internalized stigma. One could reason, based on our findings, that patients would be capable of adapting their social engagement in the workplace or other goal-directed contexts to their liking, just like they do in unstructured leisure activities, if they had the opportunity to do so. Even though engagement in paid and unpaid work is predictive of the level of social inclusion (Turner et al., 2015), a systematic review of studies of service user perspectives on work attainment identified stigma and discrimination as a crucial hindrance for individuals with psychosis (Wood and Alsawy, 2017). In a recent qualitative study of first-person accounts of social behavior, however, individuals with psychosis indicated that in addition to diminished drive, the fear of rejection sometimes linked to internalized stigma prevented them from engaging in social interactions. These factors, along with other putative mechanisms undermining engagement in the social contexts of the everyday life such as anticipatory pleasure, motivation, the willingness and ability to exert effort, warrant further exploration. The current study offers a valid method to discern the structured and unstructured social contexts by showing differential associations with lifestyle measures and clinical negative symptom severity. It is important to emphasize, however, that due to the cross-sectional design of the study, no causal relationships between lifestyle, avolition and time allocated to various social contexts could be inferred. Additionally, on a group level, the symptoms of avolition were mild, possibly due to the fact that nearly half of all patients were selected to participate based on their acutely-elevated positive symptoms and tended to have mild negative symptoms and reside in inpatient units or assisted living facilities. Another subgroup of the patients, contrastingly, tended to live independently, and endorsed relatively low 
positive as well as negative symptoms. While this patient group composition makes our findings generalizable to a broad range of community functioning and positive symptomatology, they may be less representative of groups with prominent negative symptoms or the deficit syndrome. Replication of our findings in populations further on the avolition continuum is therefore necessary.

Of note, the inclusion of sub-groups that both showed mild avolition, yet such different lifestyles might also account for the rather surprising lack of association between the two. Previous research identified avolition in addition to the positive symptoms as important disease-specific predictors of employment and real-life functioning of a large sample of patients living in the community (Galderisi et al., 2013). Despite the substantial proportion of inpatients in our sample obscuring the strength of the association between avolition and lifestyle, however, it did not affect the proportion of time patients spent in unstructured social contexts. As one of the indices of real-world social functioning, it was associated with the severity of avolition in our study.

Limitations of the present study, in addition to those listed here, have been described in detail elsewhere (Oorschot et al., 2013), and include the PANSS negative symptom items being based entirely on observation during the interview and family report, the presence of a small sample of patients with a schizoaffective disorder, and gender differences between the two samples.

With these limitations in mind, the findings of the present study support the notion that asociality of the psychotic disorder might be a manifestation of both, avolition as well as passive lifestyle. Zooming in on the contexts in which social interactions occur in the real world and real lives of individuals with psychosis appears to be a valid and promising approach for further inquiries into asociality that could lead to functional interventions designed to increase patients 'social engagement (Fowler et al., 2018). 
Funding: This work was supported by the ERC consolidator grant (ERC-2012-StG, project 309767-INTERACT) and FWO Odysseus grant to Inez Myin-Germeys. 


\section{References}

American Psychiatric Association, 2013. Diagnostic and statistical manual of mental disorders, fifth edition. American Psychiatric Association, Arlington, VA.

Andreasen, N.C., Flaum, M., Arndt, S., 1992. The comprehensive assessment of symptoms and history (cash). An instrument for assessing diagnosis and psychopathology. Arch. Gen. Psychiatry 49 (8), 615-623.

Bodapati, A.S., Herbener, E.S., 2014. The impact of social content and negative symptoms on affective ratings in schizophrenia. Psychiatry Res. 218 (1-2), 25-30.

Cohen, A.S., Minor, K.S., 2010. Emotional experience in patients with schizophrenia revisited: Metaanalysis of laboratory studies. Schizophr. Bull. 36 (1), 143-150.

Collip, D., Wigman, J.T., van Os, J., Oorschot, M., Jacobs, N., Derom, C., et al., 2014. Positive emotions from social company in women with persisting subclinical psychosis: Lessons from daily life. Acta Psychiatr. Scand. 129 (3), 202-210.

Dowd, E.C., Barch, D.M., 2010. Anhedonia and emotional experience in schizophrenia: Neural and behavioral indicators. Biol. Psychiatry 67 (10), 902-911.

Eklund, M., Leufstadius, C., Bejerholm, U., 2009. Time use among people with psychiatric disabilities: Implications for practice. Psychiatric Rehabilitation Journal 32 (3), 177-191.

Fervaha, G., Foussias, G., Agid, O., Remington, G., 2014. Motivational and neurocognitive deficits are central to the prediction of longitudinal functional outcome in schizophrenia. Acta Psychiatr. Scand. 130 (4), 290-299.

Fortunati, R., Ossola, P., Camerlengo, A., Bettini, E., De Panfilis, C., Maggini, C., et al., 2015. Anhedonia in schizophrenia: The role of subjective experiences in the emotion paradox. Eur. Psychiatry 30.

Fowler, D., Hodgekins, J., French, P., Marshall, M., Freemantle, N., McCrone, P., et al., 2018. Social recovery therapy in combination with early intervention services for enhancement of social recovery in patients with first-episode psychosis (supereden3): A single-blind, randomised controlled trial. Lancet Psychiatry 5 (1), 41-50.

Galderisi, S., Bucci, P., Mucci, A., Kirkpatrick, B., Pini, S., Rossi, A., et al., 2013. Categorical and dimensional approaches to negative symptoms of schizophrenia: Focus on long-term stability and functional outcome. Schizophr. Res. 147 (1), 157-162.

Gard, D.E., Kring, A.M., Gard, M.G., Horan, W.P., Green, M.F., 2007. Anhedonia in schizophrenia: Distinctions between anticipatory and consummatory pleasure. Schizophr. Res. 93 (1-3), 253 260.

Gard, D.E., Sanchez, A.H., Cooper, K., Fisher, M., Garrett, C., Vinogradov, S., 2014a. Do people with schizophrenia have difficulty anticipating pleasure, engaging in effortful behavior, or both? J. Abnorm. Psychol. 123 (4), 771-782.

Gard, D.E., Sanchez, A.H., Starr, J., Cooper, S., Fisher, M., Rowlands, A., et al., 2014b. Using selfdetermination theory to understand motivation deficits in schizophrenia: The 'why' of motivated behavior. Schizophr. Res. 156 (2-3), 217-222.

Gold, J.M., Strauss, G.P., Waltz, J.A., Robinson, B.M., Brown, J.K., Frank, M.J., 2013. Negative symptoms of schizophrenia are associated with abnormal effort-cost computations. Biol. Psychiatry 74 (2), 130-136.

Gold, J.M., Waltz, J.A., Frank, M.J., 2015. Effort cost computation in schizophrenia: A commentary on the recent literature. Biol. Psychiatry 78 (11), 747-753.

Heerey, E.A., Gold, J.M., 2007. Patients with schizophrenia demonstrate dissociation between affective experience and motivated behavior. J. Abnorm. Psychol. 116 (2), 268-278.

Horan, W.P., Wynn, J.K., Kring, A.M., Simons, R.F., Green, M.F., 2010. Electrophysiological correlates of emotional responding in schizophrenia. J. Abnorm. Psychol. 119 (1), 18-30.

Jang, S.K., Choi, H.I., Park, S., Jaekal, E., Lee, G.Y., Cho, Y.I., et al., 2016. A two-factor model better explains heterogeneity in negative symptoms: Evidence from the positive and negative syndrome scale. Front. Psychol. 7, 707. 
Lipskaya-Velikovsky, L., Jarus, T., Easterbrook, A., Kotler, M., 2016. Participation in daily life of people with schizophrenia in comparison to the general population. Can. J. Occup. Ther. 83 (5), 297-305.

Lui, S.S., Shi, Y.F., Au, A.C., Li, Z., Tsui, C.F., Chan, C.K., et al., 2016. Affective experience and motivated behavior in schizophrenia spectrum disorders: Evidence from clinical and nonclinical samples. Neuropsychology 30 (6), 673-684.

Marder, S.R., Galderisi, S., 2017. The current conceptualization of negative symptoms in schizophrenia. World Psychiatry 16 (1), 14-24.

McCarthy, J.M., Treadway, M.T., Bennett, M.E., Blanchard, J.J., 2016. Inefficient effort allocation and negative symptoms in individuals with schizophrenia. Schizophr. Res. 170 (2-3), 278-284.

Myin-Germeys, I., Oorschot, M., Collip, D., Lataster, J., Delespaul, P., van Os, J., 2009. Experience sampling research in psychopathology: Opening the black box of daily life. Psychol. Med. 39 (9), 1533-1547.

Olsson, A.K., Hjarthag, F., Helldin, L., 2016. Predicting real-world functional milestones in schizophrenia. Psychiatry Res. 242, 1-6.

Oorschot, M., Lataster, T., Thewissen, V., Lardinois, M., Wichers, M., van Os, J., et al., 2013. Emotional experience in negative symptoms of schizophrenia--no evidence for a generalized hedonic deficit. Schizophr. Bull. 39 (1), 217-225.

Oorschot, M., Lataster, T., Thewissen, V., Wichers, M., Myin-Germeys, I., 2012. Mobile assessment in schizophrenia: A data-driven momentary approach. Schizophr. Bull. 38 (3), 405-413.

Rocca, P., Montemagni, C., Zappia, S., Pitera, R., Sigaudo, M., Bogetto, F., 2014. Negative symptoms and everyday functioning in schizophrenia: A cross-sectional study in a real world-setting. Psychiatry Res. 218 (3), 284-289.

Strauss, G.P., 2013. The emotion paradox of anhedonia in schizophrenia: Or is it? Schizophr. Bull. 39 (2), 247-250.

Suri, G., Lavaysse, L.M., Young, G., Moodie, C., Tersakyan, A., Gross, J.J., et al., 2018. An investigation into the drivers of avolition in schizophrenia. Psychiatry Res. 261, 225-231.

Turner, N., O'Mahony, P., Hill, M., Fanning, F., Larkin, C., Waddington, J., et al., 2015. Work life after psychosis: A detailed examination. Work 51 (1), 143-152.

van der Gaag, M., Hoffman, T., Remijsen, M., Hijman, R., de Haan, L., van Meijel, B., et al., 2006. The five-factor model of the positive and negative syndrome scale ii: A ten-fold crossvalidation of a revised model. Schizophr. Res. 85 (1-3), 280-287.

Wood, L., Alsawy, S., 2017. Recovery in psychosis from a service user perspective: A systematic review and thematic synthesis of current qualitative evidence. Community Ment. Health J.

Woodward, T.S., Jung, K., Smith, G.N., Hwang, H., Barr, A.M., Procyshyn, R.M., et al., 2014. Symptom changes in five dimensions of the positive and negative syndrome scale in refractory psychosis. Eur. Arch. Psychiatry Clin. Neurosci. 264 (8), 673-682.

Xie, D.J., Lui, S.S.Y., Geng, F.L., Yang, Z.Y., Zou, Y.M., Li, Y., et al., 2017. Dissociation between affective experience and motivated behaviour in schizophrenia patients and their unaffected first-degree relatives and schizotypal individuals. Psychol. Med., 1-13.

Yan, C., Cao, Y., Zhang, Y., Song, L.L., Cheung, E.F., Chan, R.C., 2012. Trait and state positive emotional experience in schizophrenia: A meta-analysis. PLoS One 7 (7), e40672.

\section{Figure Caption:}

Figure 1: Comparison of the lifestyle breakdown of individuals with the psychotic disorder and healthy control subjects

Each segment of the pie chart represents the proportion of participants that fall within one of the four lifestyle categories. 1a) The majority of individuals with the psychotic disorder did 
not participate in any vocational activities while cohabitating with others. 1b) The vast majority of control subjects participated in vocational activities while cohabitating, and none had a lifestyle characterized by no vocational activities without cohabitation. The two groups differed significantly on the percentage of participants with lifestyle category $\mathrm{B}(\mathrm{OR}=32.42$, $z=8.64, p<0.001)$ and $\mathrm{D}(\mathrm{OR}=0.027, z=-10.75, p<0.001)$, but were comparable on category $\mathrm{C}$ lifestyle $(\mathrm{OR}=0.76, z=-0.58, p=0.565)$. $\mathrm{OR}=$ odds ratio, $z=z$-statistic, $p=p$-value. 
$1 a$

Individuals with psychotic disorder

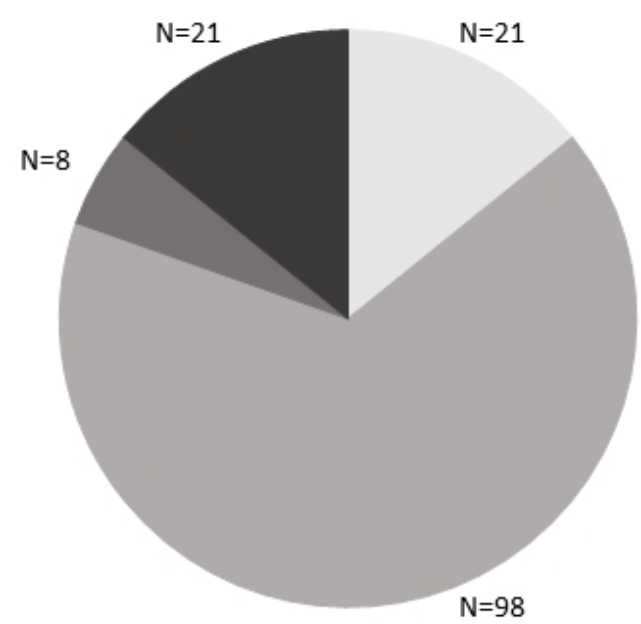

$\mathrm{A}=$ no vocational activities, no cohabitation

$\mathrm{B}=$ no vocational activities, cohabitation
$1 b$

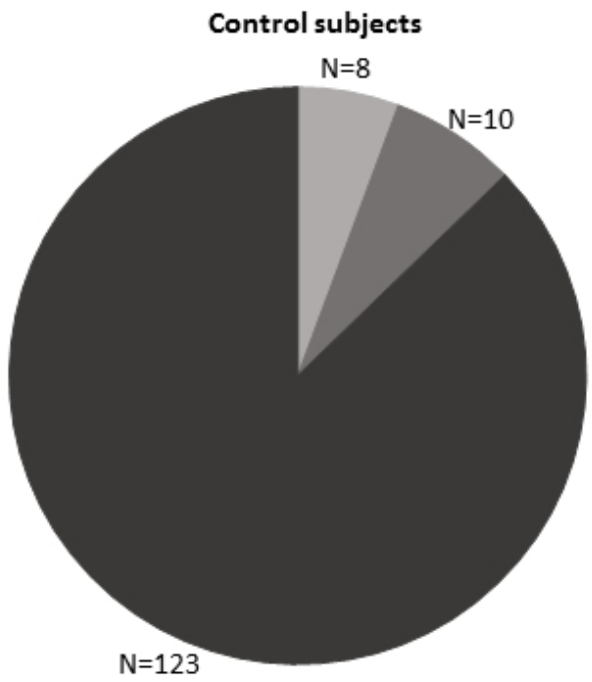

$\mathrm{C}=$ vocational activities, no cohabitation

$D=$ vocational activities, cohabitation 
Table 1: Demographic, symptom, lifestyle and experience sampling assessments

\begin{tabular}{|c|c|c|c|c|c|c|c|}
\hline \multirow[b]{2}{*}{ Gender } & \multicolumn{2}{|c|}{$\begin{array}{c}\text { Individuals with } \\
\text { psychosis } \\
N=149 \\
\end{array}$} & \multicolumn{2}{|c|}{$\begin{array}{l}\text { Healthy controls } \\
\qquad N=143\end{array}$} & \multicolumn{3}{|c|}{ Group comparison } \\
\hline & Men & Women & Men & Women & OR & $z$ & $p$ \\
\hline & 104 & 45 & 56 & 87 & 0.65 & -5.17 & $<0.001$ \\
\hline \multirow[t]{2}{*}{ Age } & $M$ & SD & $M$ & SD & B & $t$ & $p$ \\
\hline & 38.77 & 10.96 & 39.74 & 12.77 & -1.21 & -2.77 & 0.006 \\
\hline \multicolumn{8}{|l|}{ PANSS symptom scores } \\
\hline Positive symptoms & 14.82 & 8.07 & \multicolumn{2}{|c|}{-} & - & - & - \\
\hline Disorganization symptoms & 13.4 & 4.63 & \multicolumn{2}{|c|}{-} & - & - & - \\
\hline Excitement & 5.7 & 2.83 & \multicolumn{2}{|c|}{-} & - & - & - \\
\hline Emotional distress & 8.72 & 3.41 & \multicolumn{2}{|c|}{-} & - & - & - \\
\hline Expressive deficit & 6.5 & 3.45 & \multicolumn{2}{|c|}{-} & - & - & - \\
\hline Avolition symptoms & 5.13 & 2.98 & \multicolumn{2}{|c|}{ 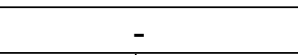 } & - & - & - \\
\hline Experience Sampling Constructs & $M$ & SD & $M$ & SD & B & $z$ & $p$ \\
\hline Mean PA & 4.38 & 1.03 & 5.28 & .72 & -.82 & -7.12 & $<0.001$ \\
\hline $\begin{array}{l}\text { Proportion of time in goal-directed } \\
\text { activities }\end{array}$ & 0.27 & 0.14 & 0.51 & 0.14 & -1.05 & -12.77 & $<0.001$ \\
\hline $\begin{array}{l}\text { Proportion of time in structured } \\
\text { social contexts }\end{array}$ & 0.31 & 0.23 & 0.47 & 0.19 & -0.74 & -5.29 & $<0.001$ \\
\hline $\begin{array}{l}\text { Proportion of time in unstructured } \\
\text { social contexts }\end{array}$ & 0.22 & 0.2 & 0.21 & 0.15 & 0.12 & 0.77 & 0.443 \\
\hline Demographic information & \multicolumn{2}{|c|}{$\%$ total sample } & \multicolumn{2}{|c|}{$\%$ total sample } & OR & $z$ & $p$ \\
\hline \multicolumn{8}{|l|}{ Civic status } \\
\hline Married & \multicolumn{2}{|c|}{8.75} & \multicolumn{2}{|c|}{59.41} & 0.07 & -7.91 & $<0.001$ \\
\hline Divorced & \multicolumn{2}{|c|}{9.95} & \multicolumn{2}{|c|}{6.14} & 1.92 & 1.51 & 0.130 \\
\hline Widow & \multicolumn{2}{|c|}{0.87} & \multicolumn{2}{|c|}{0} & - & - & - \\
\hline Cohabitation & \multicolumn{2}{|c|}{6.28} & \multicolumn{2}{|c|}{14.49} & 0.40 & -2.21 & 0.027 \\
\hline Single & \multicolumn{2}{|c|}{74.14} & \multicolumn{2}{|c|}{19.96} & 9.45 & 8.23 & $<0.001$ \\
\hline \multicolumn{8}{|l|}{ Living situation } \\
\hline Alone & \multicolumn{2}{|c|}{19.55} & \multicolumn{2}{|c|}{6.70} & 3.78 & 2.89 & 0.004 \\
\hline Partner/own family & \multirow{2}{*}{\multicolumn{2}{|c|}{$\frac{14.94}{1864}$}} & \multicolumn{2}{|c|}{79.26} & .05 & -9.69 & $<0.001$ \\
\hline Parents/other family & & & & & 1.23 & 0.64 & 0.521 \\
\hline
\end{tabular}




\begin{tabular}{|c|c|c|c|c|c|}
\hline Housemates & 4.96 & 0.64 & 8.06 & 1.95 & 0.051 \\
\hline Psychiatric institution & 40.01 & 0 & - & - & - \\
\hline Other setting & 1.25 & 0 & - & - & - \\
\hline \multicolumn{6}{|l|}{ Vocational status } \\
\hline Regular paid work & 8.58 & 72.67 & 0.03 & -9.53 & $<0.001$ \\
\hline Volunteer/sheltered work & 4.76 & 0 & - & - & - \\
\hline School/education & 6.19 & 12.49 & 1.60 & 3.17 & 0.002 \\
\hline Stay-at-home parent/partner & 0.48 & 8.82 & 0.07 & -2.49 & 0.013 \\
\hline$>3$ month sick leave & 4.21 & 0 & - & - & - \\
\hline Unfit for work & 63.47 & 2.28 & 82.1 & 7.25 & $<0.001$ \\
\hline Unemployed & 11.07 & 2.10 & 6.41 & 2.92 & 0.003 \\
\hline Retired & 0 & 0.84 & - & - & - \\
\hline Other & 1.24 & 0.80 & 1.93 & 0.54 & 0.592 \\
\hline
\end{tabular}


Table 2: The association between lifestyle and proportion of time spent in social contexts

\begin{tabular}{|c|c|c|c|c|c|}
\hline \multicolumn{7}{|c|}{ Structured social contexts } \\
\hline Lifestyle & Contrast & $\boldsymbol{Z}$ & $\boldsymbol{p}^{*}$ & $\mathbf{9 5 \%}$ Conf. Interval \\
\hline A vs. B & 0.87 & 2.90 & 0.022 & 0.08 & 1.67 \\
\hline A vs. C & 0.22 & 0.43 & 1.000 & -1.14 & 1.59 \\
\hline A vs. D & 1.86 & 4.85 & $<0.001$ & 0.85 & 2.87 \\
\hline B vs. C & -0.65 & -1.42 & 0.936 & -1.86 & 0.56 \\
\hline D vs. B & 0.99 & 3.31 & 0.006 & 0.2 & 1.78 \\
\hline D vs. C & 1.64 & 3.17 & 0.009 & 0.28 & 3.0 \\
\hline \multicolumn{7}{|c|}{ Unstructured social contexts } \\
\hline A vs. B & 0.93 & 2.99 & 0.017 & 0.11 & 1.76 \\
\hline A vs. C & 0.38 & 0.71 & 1.000 & -1.02 & 1.76 \\
\hline A vs. D & 1.54 & 3.88 & 0.001 & 0.45 & 2.6 \\
\hline B vs. C & -0.56 & -1.21 & 1.000 & -1.78 & 0.66 \\
\hline D vs. B & 0.61 & 1.99 & 0.280 & -0.2 & 1.42 \\
\hline D vs. C & 1.17 & 2.23 & 0.154 & -0.21 & 2.55 \\
\hline
\end{tabular}

${ }^{*}$ Bonferroni-corrected for multiple comparisons

$A=$ no vocational activities, lives alone; $B=$ no vocational activities, lives with others; $C=$ vocational activities, lives alone; $D=$ vocational activities, lives with others. $z=z$-statistic, $p=p$-value. 
Table 3: The associations between social contexts and positive affect (i.e. hedonic experience) for individuals with psychosis, healthy controls, and between-group comparisons.

\begin{tabular}{|c|c|c|c|c|c|c|c|c|c|c|c|c|c|c|c|}
\hline & \multicolumn{15}{|c|}{ Hedonic experience } \\
\hline & \multicolumn{5}{|c|}{ Individuals with psychosis } & \multicolumn{5}{|c|}{ Healthy controls } & \multicolumn{5}{|c|}{ Group difference } \\
\hline Social context & $\mathbf{B}$ & $\boldsymbol{z}$ & $p$ & $\begin{array}{r}95 \% \\
\text { Int }\end{array}$ & $\begin{array}{l}\text { Conf. } \\
\text { val }\end{array}$ & B & $\boldsymbol{z}$ & $p$ & $\begin{array}{r}95 \% \\
\text { Int }\end{array}$ & $\begin{array}{l}\text { onf. } \\
\text { al }\end{array}$ & B & $z$ & $p$ & $\begin{array}{r}95 \% \\
\text { Inte }\end{array}$ & $\begin{array}{l}\text { nff. } \\
\text { al }\end{array}$ \\
\hline Structured & -0.03 & -0.56 & 0.578 & -0.13 & 0.07 & 0.01 & 0.44 & 0.661 & -0.04 & 0.07 & -0.04 & -0.58 & 0.565 & -0.16 & 0.09 \\
\hline Unstructured & 0.2 & 3.52 & $<0.001$ & 0.09 & 0.31 & 0.24 & 5.54 & $<0.001$ & 0.15 & 0.32 & -0.04 & -0.5 & 0.617 & -0.2 & 0.12 \\
\hline
\end{tabular}

$\mathrm{B}=$ beta coefficient, $z=z$-statistic, $p=p$-value 
Supplementary Table S1: The association between the avolition symptom score and lifestyle

\begin{tabular}{|c|c|c|c|c|c|}
\hline Lifestyle & RRR & $\boldsymbol{z}$ & $\boldsymbol{p}^{*}$ & \multicolumn{2}{|c|}{ 95\% Conf. Interval } \\
\hline $\mathrm{A}$ & 1.36 & 1.27 & 0.203 & 0.85 & 2.2 \\
\hline $\mathrm{C}$ & 0.46 & -1.09 & 0.278 & 0.12 & 1.86 \\
\hline $\mathrm{D}$ & 0.67 & -0.88 & 0.377 & 0.28 & 1.62 \\
\hline
\end{tabular}

*Bonferroni-corrected for multiple comparisons

$\dagger \mathrm{B}$ lifestyle was used as the reference category, as it was the most common one ( $N=98$ patients). $\mathrm{A}=$ no vocational activities, no cohabitation; $\mathrm{B}=$ no vocational activities, cohabitation; $\mathrm{C}=$ vocational activities, no cohabitation; $\mathrm{D}=$ vocational activities, cohabitation. $\mathrm{RRR}=$ relative risk ratio for a one standard deviation (SD) increase in PANSS avolition score for having a lifestyle A, $\mathrm{C}$ or D versus lifestyle B, respectively. For instance, given a one SD increase in avolition score, the relative risk of having lifestyle category A versus B would be expected to increase by a factor of 1.36. $z=z$-statistic, $p=p$-value. 